\title{
Optimal Distraction Force for Evaluating Tibiofemoral Joint Gaps in Posterior Stabilized Total Knee Arthroplasty
}

\author{
Yasushi Oshima, Norishige Iizawa, Shinro Takai and Tokifumi Majima \\ Department of Orthopaedic Surgery, Nippon Medical School, Tokyo, Japan
}

\begin{abstract}
Background: Obtaining well-balanced soft tissues is important to achieve natural knee kinematics after total knee arthroplasty (TKA). In conventional procedures, soft tissue balance is evaluated with spacer blocks or lamina spreaders. However, the evaluation depends on the surgeons' experience and is not quantitative. This study aims to measure the mechanical properties of knee soft tissue with a new ligament balancer and to determine the optimal distraction force for evaluating tibiofemoral joint gaps in TKA.
\end{abstract}

Methods: This study included 30 consecutive patients with medial knee osteoarthritis who were scheduled to undergo posterior stabilized TKA. The mean age of patients was $73 \pm 9.6$ years at the time of surgery, and the mean hip-knee-ankle angle was $13.1 \pm 6.5^{\circ}$ in varus. After distal femoral and proximal tibial resections, the tibiofemoral joint gaps under several distraction forces were measured in extension and at $90^{\circ}$ flexion. The load-displacement curves in extension and flexion were drawn with these data, and the stability range, which was defined as the shift range from the toe region to the linear region in the curves, was calculated.

Results: The stability ranges were 160 Newtons (N) in extension and $140 \mathrm{~N}$ in flexion.

Conclusions: These displacement forces were considered optimal for evaluating tibiofemoral joint gaps during surgery and ensuring knee stability after TKA. (J Nippon Med Sch 2021; 88: 361-366)

Key words: posterior stabilized total knee arthroplasty (PS-TKA), tibiofemoral joint gap, ligament balancer, modified gap-balancing technique, load-displacement curve

\section{Introduction}

Total knee arthroplasty (TKA) is a successful treatment option for severe osteoarthritis of the knee (knee OA). It eliminates pain, increases range of motion (ROM), and improves quality of life ${ }^{1}$. With developments in basic anatomical research, surgical procedures, and the technology of artificial prostheses, the longevity of recent TKAs is estimated to be more than 15 years ${ }^{2}$. Consequently, the number of TKA cases has dramatically increased in aging societies and will reach approximately 3 million per year in the United States by $2030^{3}$. Nevertheless, more than $15 \%$ of patients reported being less than fully satisfied, particularly when participating in activities involving stairs or sports ${ }^{4}$.

Previously, polyethylene wear of the tibial insert was reported to be a critical factor in TKA failure ${ }^{5}$. However, in the past few decades, with improved material quality, polyethylene wear is no longer the primary cause of failure $^{6}$. The current major causes of failure were reported to be aseptic loosening, infection, instability, and malalignment and malrotation ${ }^{7,8}$. Most of these factors have diminished with decreases in technical errors.

Successful TKA requires knowledge of the precise surgical procedure to obtain accurate knee alignment and of the well-balanced soft tissues around the knee. Conventionally, extramedullary and intramedullary rod techniques have been used for accurate knee alignment with precise bone resections. Preoperative radiographs and CT images are also useful for surgical planning. We previously developed a CT template technique that used pre-

Correspondence to Yasushi Oshima, Department of Orthopaedic Surgery, Nippon Medical School, 1-1-5 Sendagi, Bunkyo-ku,

Tokyo 113-8603, Japan

E-mail: y-oshima@nms.ac.jp

https://doi.org/10.1272/jnms.JNMS.2021_88-519

Journal Website (https://www.nms.ac.jp/sh/jnms/) 
1

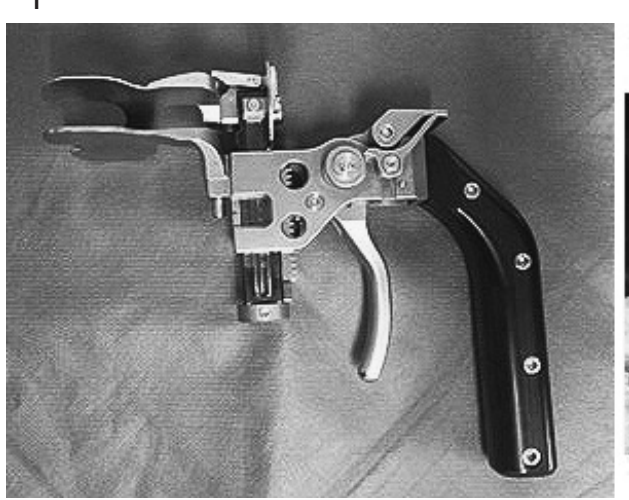

2

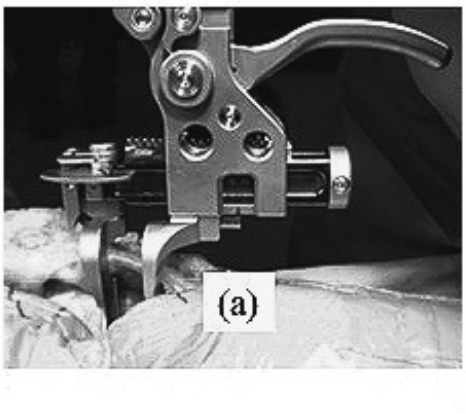

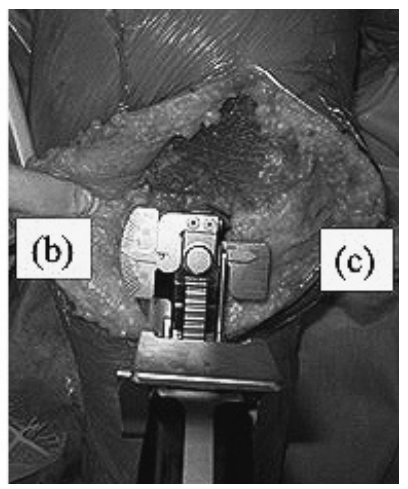

Fig. 1 A spring-loaded ligament balancer (1) and the knee in extension (2) and flexion (3). The scales show the tibiofemoral center gap in $\mathrm{mm}(\mathrm{a})$, the angle between the femoral and proximal tibial surfaces in degrees (b), and the distraction force in $\mathrm{N}$ (c).

operative CT images to improve the rotational alignment of the knee'. Computer technologies, i.e., computer navigation surgeries and robotic surgeries, have recently been introduced in TKA, and these technologies have improved bone resection accuracy ${ }^{10,11}$.

To adjust knee balancing, soft tissues are released to create rectangular and symmetrical extension and flexion gaps in the gap-balancing technique-a prerequisite for stable knee kinematics in $\mathrm{TKA}^{12}$. Medial soft tissue release should start from the deep medial collateral ligament (dMCL) and superficial medial collateral ligament (sMCL), followed by the posterior oblique ligament, semimembranosus muscle, and pes anserine tendons ${ }^{13}$. Then, soft tissue balance is evaluated with spacer blocks or lamina spreaders ${ }^{14}$. However, assessment of knee balancing is based on the surgeons' preference, and a procedure to quantitatively evaluate the appropriate soft tissue balance has not been established ${ }^{15,16}$. Ligament balancers were created to resolve this; however, the amount of distraction force required to obtain knee stability has not been determined. Therefore, current TKA outcomes still depend on the surgeons' preference, which explains why TKA outcomes appear to be worse for low-volume surgeons and centers than for high-volume surgeons and centers ${ }^{17}$.

We hypothesized that obtaining a well-balanced knee post-TKA requires determination of the appropriate distraction force for evaluating the tibiofemoral joint gap. In this study, we developed a new spring-loaded ligament balancer (Umihira Co. Ltd., Kyoto, Japan) that uses several distraction forces to measure simultaneously the distance of the tibiofemoral center gap and angle between the femoral and proximal tibial surfaces (Fig. 1). This study attempted to measure the mechanical properties of knee soft tissues with this ligament balancer and to determine the optimal distraction force exertable for evaluating tibiofemoral joint gaps, to ensure knee stability post-TKA.

\section{Patients and Methods}

This study included Japanese patients with radiographic primary varus knee OA who were consecutively scheduled for primary TKA with posterior stabilized (PS) implants at our center between September 2017 and August 2018. Patients with severe knee deformity [i.e., flexion contracture greater than $30^{\circ}$; lateral femorotibial angle (FTA), also known as anatomical axis, greater than $200^{\circ}$; or a bone defect requiring metal augmentation], valgus knee deformity, past history of knee injuries or operative procedures for the knee, or rheumatoid arthritis or inflammatory arthritis were excluded. The study was approved by the relevant institutional review board (No. 29-09-821), and the enrolled patients provided informed consent to participate in the study.

\section{Surgical Procedures}

All TKA operations were performed by the same senior surgeon using the modified gap balancing technique. The surgical procedure was performed with infiltration of an air tourniquet under general anesthesia. An incision was made in the mid-longitudinal skin, and knee arthrotomy was performed using the medial parapatellar approach.

After the patella was laterally retracted without eversion, the medial joint capsule was subperiosteally separated $10 \mathrm{~mm}$ caudally from the tibial joint line and from the anteromedial part until reaching the anterior edge of the dMCL; the dMCL itself was left intact. Femoral and tibial osteophytes were removed, after which the anterior 
Table

\begin{tabular}{ll}
\hline Total number of patients & 30 \\
Men & 10 \\
Women & 20 \\
Mean age at surgery & $73 \pm 9.6$ years $(50-85$ years $)$ \\
Body mass index (BMI) & $27.0 \pm 4.9(21.7-33.3)$ \\
Hip-knee-ankle angle & $13.1^{\circ} \pm 6.5^{\circ}\left(5^{\circ}-20^{\circ}\right)$ in varus deformity \\
\hline
\end{tabular}

1

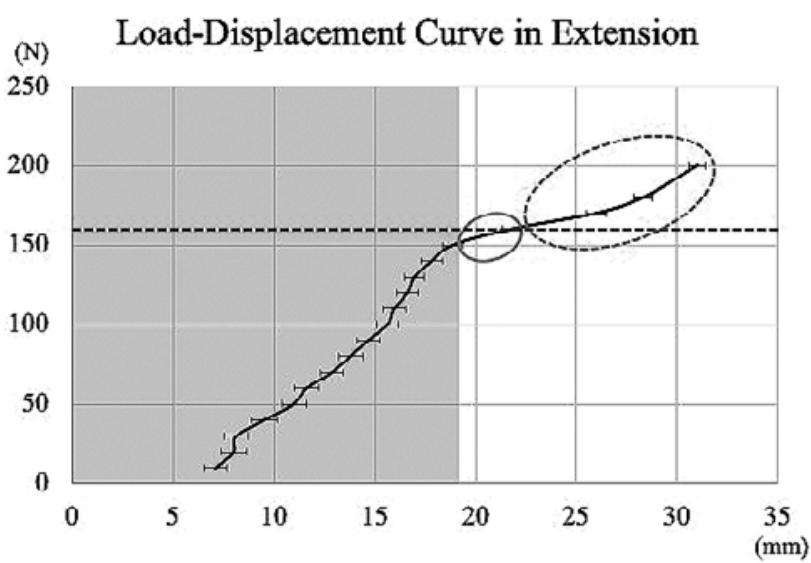

2

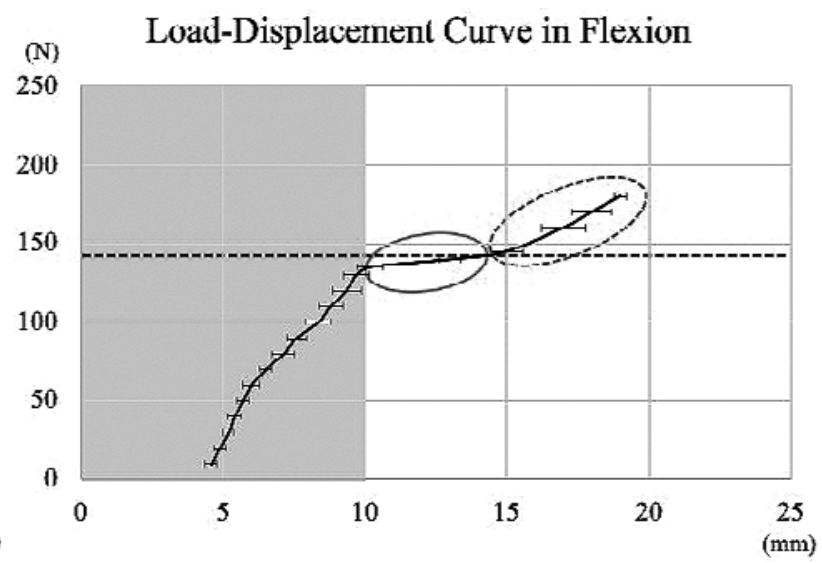

Fig. 2 The load-displacement curves in extension (1) and flexion (2). The solid oval line indicates the toe region, and the dotted oval line indicates the linear region.

The intersections of these regions are recognized as the stability ranges (the dotted straight lines), which were $160 \pm$ $12 \mathrm{~N}$ in extension and $140 \pm 10 \mathrm{~N}$ in flexion.

cruciate ligament (ACL) and posterior cruciate ligament (PCL) were removed.

The distal femur was resected perpendicular to the mechanical axis with the intramedullary femoral rod technique. The bone was resected with $9 \mathrm{~mm}$ thickness from the joint line, as was the case for the femoral component. Similarly, the proximal tibia was resected at $10 \mathrm{~mm}$, with the same thickness as that of the tibial component, from the lateral tibial joint line by using extramedullary rod technique, perpendicular to the tibial shaft axis.

After the bone resections, the ligament balancer was applied to measure the gap variation with the distraction forces in extension and at $90^{\circ}$ flexion (Fig. 1). The ligament balancer is a spring-loaded center paddle device and is graduated in 10-Newton (N) intervals. The maximum load is $200 \mathrm{~N}$, which is indicated on a calibrated scale. It also measures the center gap, i.e., the distance between the center of the distal femur and proximal tibia. The minimal unit of this device is $1 \mathrm{~mm}$. The angle between the distal femur and proximal tibia was measured simultaneously. The minimum angle of this device is $1^{\circ}$.

The center gap and the angle between the femoral and tibial surfaces in full extension and at $90^{\circ}$ flexion were measured by applying a distraction force up to $200 \mathrm{~N}$. Thereafter, the TKA procedure was performed as usual.

\section{Load-Displacement Curve}

The load-displacement curves were drawn with the distraction force and joint gap in extension and flexion. Then, the approximate straight lines of the toe region and linear region were calculated with the least squares method. In this study, the stability range was defined as the shift range from the toe region to the linear region, and the appropriate load for evaluating gaps in obtaining knee stability post-TKA was defined as the intersection of these lines.

\section{Results}

This study included 30 patients: 10 men and 20 women. The patient demographics are shown in the Table. All patients had grade IV OA knees on the KellgrenLawrence classification. The assessments were made by using preoperative anteroposterior knee radiographs ${ }^{18}$. The load-displacement curves showed that the toe region and linear region were both in extension and in flexion (Fig. 2-1 and 2). 
In total, the thickness of the bone resections was 19 $\mathrm{mm}$, with the sum of the femur and tibia in extension, and $10 \mathrm{~mm}$, with only the tibia in flexion. As displacement load may have been theoretically insufficient under the $19 \mathrm{~mm}$ in extension and $10 \mathrm{~mm}$ in flexion, these ranges were not involved in the toe region, and the approximate straight lines were calculated with ranges higher than $19 \mathrm{~mm}$ in extension and $10 \mathrm{~mm}$ in flexion. The results showed stiffness scores of $6.4 \mathrm{~N} / \mathrm{mm}$ and 9.8 $\mathrm{N} / \mathrm{mm}$ in extension and flexion, respectively. The intersections of these lines were calculated as $160 \pm 12 \mathrm{~N}$ in extension and $140 \pm 10 \mathrm{~N}$ in flexion.

\section{Discussion}

Tibiofemoral joint gap variations in extension and flexion were measured during PS-TKA with a newly developed ligament balancer. To obtain knee stability post-TKA, the optimal distraction forces to evaluate the gaps were 160 $\mathrm{N}$ in extension and $140 \mathrm{~N}$ in flexion.

Measurement of the tibiofemoral joint gap during TKA is important in achieving well-balanced knee kinematics. Conventionally, soft tissues have been released stepwise to create rectangular and symmetric gaps ${ }^{12}$. Then, spacer blocks or lamina spreaders have been used to measure soft tissue balance. The steel rod test was developed to evaluate tension in collateral ligaments ${ }^{19}$. However, obtaining perfect rectangular and symmetric gaps is technically difficult ${ }^{20}$. Moreover, assessment of knee balance remained subjective and dependent on the surgeons' experience. Recently, spacer blocks are not used to estimate the accuracy of the extension gap, because of their flat shape and lack of posterior condylar offset of the femoral component ${ }^{21}$. Thus, it is an unreliable technique for accurate evaluation of the joint gap.

In contrast to traditional soft tissue releases, current practice has shifted to the importance of medial soft tissue conservation to preserve physiological natural knee kinematics post-TKA, because physiological knee kinematics exhibit lateral pseudolaxity ${ }^{22}$. To preserve kinematics, trapezoidal and asymmetric gaps that were approximately $3 \mathrm{~mm}$ thicker laterally and in flexion were considered tolerable ${ }^{20,23,24}$. However, there is no consensus on how much ligamentous laxity or asymmetry is acceptable in gaps without sacrificing joint stability; therefore, adequate ligament balancing still needs to be understood.

To measure accurate gaps, ligament balancers have been developed by applying a torque wrench or spring. Torque is defined as the rotational equivalent of linear force, expressed in Newton meters $(\mathrm{N} \cdot \mathrm{m})$. Because torque force must be converted into distraction force, the force of the spring, in Newtons $(\mathrm{N})$, is considered a more appropriate fit for ligament balancers. Thus, we developed a new balancer that can simultaneously measure the center gap and angle between the femoral and tibial surfaces, under different distraction forces with the spring. However, when using a spring-loaded ligament balancer, Matsui et al. applied a force of $120 \mathrm{~N}$ in both gaps, whereas Ferreira et al. applied $100 \mathrm{~N}$ upon extension and $80 \mathrm{~N}$ upon flexion ${ }^{25,26}$. As the soft tissue balance has been reported to change under these varying forces ${ }^{27}$, it is necessary to find the optimal force for evaluating joint gaps.

Ligaments function as a crimp that provides slight longitudinal elongation without fibrous damage, controls tension, and acts as a shock absorber ${ }^{28}$. When mechanical force is applied to the ligament from the lower region, the shape of the ligament changes as the load increases, and this variation is illustrated by a bimodal loaddeformation curve ${ }^{29}$. This curve involves two main parts - the toe and linear regions-which indicate the slopes for ligament laxity and stiffness, respectively. When all soft tissues surrounding the knee, including ligaments, tendons, synovial membranes, and joint capsule, are regarded as a mass of soft tissues, this mass is considered to demonstrate a feature similar to the ligaments. Consequently, the knee involving all soft tissues exhibited toe and linear regions, as we expected.

Mechanical loads on knee joints have been previously studied. Mechanical forces on the ACL were typically between $150 \mathrm{~N}$ and $300 \mathrm{~N}$ during normal walking, whereas ACL forces were approximately $300 \mathrm{~N}$ during droplandings. As the maximum strength of a normal healthy ACL was reported to be $2,200 \mathrm{~N}^{30}$, general daily activities required only $10 \%$ to $15 \%$ of $\mathrm{ACL}$ maximum strength. Because the normal healthy strength of the PCL is approximately twice that of the $\mathrm{ACL}^{31}$, general loads on the PCL are lower than the maximum strength. Therefore, mechanical forces on the knee in most daily activities are lower than the knee strength and within the range of the toe region $^{32}$. As tolerance against mechanical forces on the knee is greater than that on the toe region, and a similar tolerance is considered to be necessary in post-TKA, we defined the knee stability range as the transition point from the toe region to the linear region. Our findings indicate that $160 \mathrm{~N}$ in extension and $140 \mathrm{~N}$ in flexion are the optimal distraction forces for evaluating gaps in PSTKA.

This study has some limitations. The sample size was small, and associations of knee stability with clinical out- 
comes were not evaluated. There might be sex differences in soft tissue stability. However, we noted no difference in the stability range between men and women in this study. Moreover, associations between severity of varus deformity, preoperative knee ROM, and stiffness of soft tissue were not investigated. We performed PS-TKA by applying present distraction forces to evaluate the gaps, and clinical outcomes were acceptable. Thus, the findings appear reasonable. However, future studies of clinical outcomes should be larger and have longer follow-up durations.

\section{Clinical Relevance}

Our findings suggest that the present modified gap technique is appropriate for evaluating gaps in PS-TKA.

This is our current TKA procedures. After knee arthrotomy, the medial joint capsule is released, but the dMCL is left intact. Following that, the distal femur is resected with the same thickness as the component. The proximal tibia is also resected from the lateral joint line with the same thickness as the component in the varus knee. After this, a distraction force of $160 \mathrm{~N}$ is applied upon extension, and the angle between the distal femoral surface and proximal tibial surface is measured with a ligament balancer. If the angle is greater than $3^{\circ}$, medial soft tissues, i.e., dMCL, are released, or reduction osteotomy of the medial tibia is performed by applying a tibial tray that is one size smaller. With these procedures, the angle could be adjusted in most cases at our center. Then, a $140-\mathrm{N}$ force is applied upon flexion, and the femoral rotation angle is measured. At this time, the flexion gap is confirmed to be $1 \mathrm{~mm}$ to $2 \mathrm{~mm}$ wider than the extension gap, and the posterior femoral condyle is resected. If the joint gaps or the femoral rotational angle is much different than expected, it is better to calculate the stability range by considering individual variation and apply the appropriate individual force rather than the standard forces.

\section{Conclusions}

Tibiofemoral joint gaps were measured with a newly developed ligament balancer, and knee stability ranges were calculated in extension and flexion. The optimal force to evaluate tibiofemoral joint gaps is $160 \mathrm{~N}$ in extension and $140 \mathrm{~N}$ in flexion during PS-TKA.

Conflict of Interest: The authors declare no conflict of interest.

\section{References}

1. Carr AJ, Robertsson O, Graves S, et al. Knee replacement. Lancet. 2012 Apr 7;379(9823):1331-40.

2. Gøthesen O, Espehaug B, Havelin L, et al. Survival rates and causes of revision in cemented primary total knee replacement: a report from the Norwegian Arthroplasty Register 1994-2009. Bone Joint J. 2013 May;95-B(5):636-42.

3. Kurtz SM, Ong KL, Lau E, Bozic KJ. Impact of the economic downturn on total joint replacement demand in the United States: updated projections to 2021. J Bone Joint Surg Am. 2014;96:624-30.

4. Scott CE, Howie CR, MacDonald D, Biant LC. Predicting dissatisfaction following total knee replacement: a prospective study of 1217 patients. J Bone Joint Surg Br. 2010; 92:1253-8.

5. Sharkey PF, Hozack WJ, Rothman RH, Shastri S, Jacoby SM. Why are total knee arthroplasties failing today? Clin Orthop Relat Res. 2002 Nov;(404):7-13.

6. Sharkey PF, Lichstein PM, Shen C, Tokarski AT, Parvizi J. Why are total knee arthroplasties failing today--has anything changed after 10 years? J Arthroplasty. 2014 Sep;29 (9):1774-8.

7. Pitta M, Esposito CI, Li Z, Lee YY, Wright TM, Padgett DE. Failure after modern total knee arthroplasty: A prospective study of 18,065 knees. J Arthroplast. 2018;33:40714.

8. Thiele K, Perka C, Matziolis G, Mayr HO, Sostheim M, Hube R. Current failure mechanisms after knee arthroplasty have changed: polyethylene wear is less common in revision surgery. J Bone Joint Surg Am. 2015;97:715-20.

9. Oshima Y, Iizawa N, Kataoka T, Majima T, Takai S. A computed-tomography-scan-based template to place the femoral component in accurate rotation with respect to the surgical epicondylar axis in total knee arthroplasty. Knee. 2018 Jan;25(1):195-202.

10. Sires JD, Craik JD, Wilson CJ. Accuracy of bone resection in MAKO total knee robotic-assisted surgery. J Knee Surg. 2021 Jun;34(7):745-8.

11. Zambianchi F, Luyckx T, Victor J, Digennaro V, Giorgini A, Catani F. How to improve femoral component rotational alignment in computer-assisted TKA. Knee Surg Sports Traumatol Arthrosc. 2014 Aug;22(8):1805-11.

12. Sheth NP, Husain A, Nelson CL. Surgical techniques for total knee arthroplasty: Measured resection, gap balancing, and hybrid. J Am Acad Orthop Surg. 2017 Jul;25(7): 499-508.

13. Clayton ML, Thompson TR, Mack RP. Correction of alignment deformities during total knee arthroplasties: staged soft-tissue releases. Clin Orthop Relat Res. 1986;202:11724.

14. Daines BK, Dennis DA. Gap balancing vs. measured resection technique in total knee arthroplasty. Clin Orthop Surg. 2014 Mar;6(1):1-8.

15. Fetto JF, Hadley S, Leffers KJ, Leslie CJ, Schwarzkopf R. Electronic measurement of soft-tissue balancing reduces lateral releases in total knee arthroplasty. Bull NYU Hosp Jt Dis. 2011;69(4):285-8.

16. Joseph J, Simpson PM, Whitehouse SL, English HW, Donnelly WJ. The use of navigation to achieve soft tissue balance in total knee arthroplasty - a randomized clinical study. Knee. 2013 Dec;20(6):401-6.

17. Kazarian GS, Lawrie CM, Barrack TN, et al. The impact of surgeon volume and training status on implant alignment in total knee arthroplasty. J Bone Joint Surg Am. 2019 Oct 2;101(19):1713-23. 
18. Kellgren JH, Lawrence JS. Radiological assessment of osteo-arthrosis. Ann Rheum Dis. 1957;16:494-502.

19. Ewald FC. Leg lift technique for simultaneous femoral, tibial and patella prosthetic cementing, rule of "no thumb" for patellar tracking and "steel rod rule" for ligament tension. Tech Orthop. 1991;6:44-6.

20. Griffin FM, Insall JN, Scuderi GR. Accuracy of soft tissue balancing in total knee arthroplasty. J Arthroplast. 2000; 15:970-3.

21. Jhurani A, Agarwal P, Aswal M, Meena I, Srivastava M, Sheth NP. Do spacer blocks accurately estimate deformity correction and gap balance in total knee arthroplasty? A prospective study with computer navigation. Knee. 2020 Jan;27(1):214-20.

22. Risitano S, Indelli PF. Is "symmetric" gap balancing still the gold standard in primary total knee arthroplasty? Ann Transl Med. 2017;5:325.

23. Aunan E, Kibsgård T, Clarke-Jenssen J, Röhrl SM. A new method to measure ligament balancing in total knee arthroplasty: laxity measurements in 100 knees. Arch Orthop Trauma Surg. 2012;132:1173-81.

24. In Y, Kim SJ, Kim JM, Woo YK, Choi NY, Kang JW. Agreements between different methods of gap balance estimation in cruciate-retaining total knee arthroplasty. Knee Surg Sports Traumatol Arthrosc. 2009;17:60-4.

25. Ferreira MC, Franciozi CES, Kubota MS, Priore RD, Ingham SJM, Abdalla RJ. Is the use of spreaders an accurate method for ligament balancing? J Arthroplast. 2017;327: 2262-7.

26. Matsui $Y$, Nakagawa S, Minoda Y, Mizokawa S, Tokuhara $Y$, Kadoya Y. Joint gap measurement in total knee arthroplasty using a tensor device with the same articulating surface as the prosthesis. Arch Orthop Trauma Surg. 2014 May;134(5):699-705.

27. Nagai K, Muratsu H, Matsumoto T, Miya H, Kuroda R, Kurosaka M. Soft tissue balance changes depending on joint distraction force in total knee arthroplasty. J Arthroplasty. 2014 Mar;29(3):520-4.

28. Amiel D, Billings E Jr, Akeson WH. Chapter 5, Ligament Structure, Chemistry, and Physiology. In: Daniel DM, Akeson WH, O'Connor JJ, editors. Knee Ligaments: Structure, Function, Injury, and Repair: Raven Press; 1990. p. 77-91.

29. Frank C, Woo S, Andriacchi T, et al. Chapter 2, Normal ligament: Structure, function, and composition. In: Woo SL-Y, Buckwalter JA, editors. Injury and repair of the muscloskeletal soft tissues: Am Acad Orthop Surg, Park Ridge; 1987. p. 45-101.

30. Woo SL, Hollis JM, Adams DJ, Lyon RM, Takai S. Tensile properties of the human femur-anterior cruciate ligamenttibia complex. The effects of specimen age and orientation. Am J Sports Med. 1991 May-Jun;19(3):217-25.

31. Race A, Amis AA. The mechanical properties of the two bundles of the human posterior cruciate ligament. J Biomech. 1994 Jan;27(1):13-24.

32. Escamilla RF, Zheng N, MacLeod TD, et al. Cruciate ligament tensile forces during the forward and side lunge. Clin Biomech. 2010 Mar;25(3):213-21.

(Received, September 28, 2020)

(Accepted, December 7, 2020)

(J-STAGE Advance Publication, January 19, 2021)

Journal of Nippon Medical School has adopted the Creative Commons Attribution-NonCommercial-NoDerivatives 4.0 International License (https://creativecommons.org/licenses/by-nc-nd/4.0/) for this article. The Medical Association of Nippon Medical School remains the copyright holder of all articles. Anyone may download, reuse, copy, reprint, or distribute articles for non-profit purposes under this license, on condition that the authors of the articles are properly credited. 